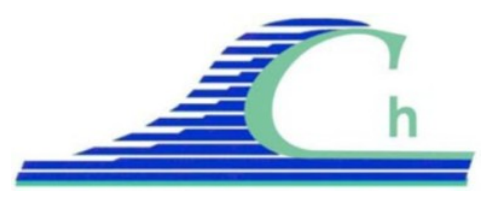

XII ${ }^{\text {èmes }}$ Journées Nationales Génie Côtier - Génie Civil

Cherbourg, $12-14$ juin 2012

DOI:10.5150/jngcgc.2012.030-G C Editions Paralia CFL

disponible en ligne - http://www.paralia.fr - available online

\title{
Modèle minimal pour les dunes transverses
}

\author{
Lucie GUIGNIER ${ }^{1}$, Alexandre VALANCE ${ }^{1}$, Dimitri LAGUE ${ }^{2}$
}

1. Université de Rennes 1, Institut de Physique de Rennes, UMR 6251 CNRS, 263 av. Général Leclerc 35042 Rennes CEDEX, France.

lucie.guignier@univ-rennes1.fr ; alexandre.valance@univ-rennes1.fr

2. Université de Rennes 1, Géosciences Rennes, UMR 6118 CNRS,

263 av. Général Leclerc 35042 Rennes CEDEX, France.

dimitri.lague@univ-rennes1.fr

\section{Résumé :}

Nous présentons ici un modèle minimal pour décrire la stabilité des dunes transverses. Ce modèle qui s'inspire de celui proposé par NIIYA et al. (2010) est basé sur une représentation simplifiée d'une dune transverse. Celle-ci est décrite par une succession de sections longitudinales qui interagissent entre elles par l'intermédiaire de flux sédimentaires latéraux. Les coupes longitudinales sont définies à partir de caractéristiques géométriques simples. La position et la hauteur de leur sommet suffisent ainsi à les caractériser entièrement. Un bilan des flux sédimentaires dans la direction longitudinale et latérale permet d'obtenir un système de deux équations couplées décrivant l'évolution spatio-temporelle de la position et de la hauteur de la ligne de crête. Ce modèle nous permet de conduire une analyse de stabilité linéaire d'une dune transverse rectiligne et d'identifier les processus physiques susceptibles de la déstabiliser au profit d'une dune sinueuse ou de la fragmenter en une multitude de dunes plus petites. Nous avons identifié deux paramètres importants dans le processus de stabilité : le taux de capture de sédiment par la face d'avalanche et les coefficients de diffusion transverses. Ce modèle simple constitue un outil intéressant pour étudier la dynamique complexe de dunes 3D.

\section{Mots-clés :}

Dunes transverses - Sédiments - Modélisation - Analyse de stabilité linéaire

\section{Introduction}

Un lit sableux, plat, horizontal, et soumis au cisaillement d'un fluide (air et eau) est intrinsèquement instable et laissera place à des structures sableuses qui présentent en général un agencement spatial régulier et que l'on appelle dunes. Elles peuvent avoir des morphologies très diverses selon l'apport en sédiment et la complexité de l'écoulement (e.g., barkhanes, dunes transverses ou étoiles). Celles-ci peuvent se former aussi bien dans les déserts de sable sous l'action du vent qu'au fond des océans ou des rivières sous l'action de courants hydrodynamiques. Le mécanisme d'instabilité primaire donnant naissance aux dunes est maintenant bien connu (RICHARDS, 1980 ; 
KROY et al., 2002 ; VALANCE, 2005) mais la dynamique ultérieure aux temps longs est encore très mal comprise du fait des fortes non-linéarités qui apparaissent dans l'écoulement et les processus de transport.

Nous intéressons ici à la stabilité des dunes éoliennes transverses se mouvant sur un sol plat non-érodable sous l'action d'un écoulement unidirectionnel d'intensité constante. Des simulations récentes basées sur la résolution complète des équations hydrodynamiques couplées à un modèle de transport de sédiment ont montré qu'une dune transverse rectiligne est intrinsèquement instable, devient sinueuse puis se disloque pour former des barkhanes (PARTELI et al., 2011). Cette approche numérique est une opération très consommatrice en temps de calcul et ne permet pas d'identifier aisément les mécanismes physiques pertinents responsables de cette instabilité. Pour cela, nous avons développé une autre approche basée sur une description simplifiée des processus mises en jeu.

L'approche que nous proposons s'inspire fortement du modèle de Niiya, Awasu et Nishimori (NIIYA et al., 2010). Elle consiste à décomposer une dune transverse en une succession de coupes transversales (i.e., des dunes bidimensionnelles) qui interagissent entre elles par l'intermédiaire de flux sédimentaires latéraux. Ce faisant, il est possible de décrire l'évolution d'une dune transverse par un simple système d'équations de type convection-diffusion pour la position et la hauteur de la ligne de crête.

\section{Modèle}

\subsection{Description du modèle}

Le modèle traite le cas d'une dune transverse qui évolue sur fond non-érodable dans le cas d'un apport de sédiment contrôlé. L’hypothèse centrale du modèle est de considérer que la coupe transversale d'une dune présente toujours la même géométrie. La pente amont et la pente aval sont supposées constantes et caractérisées respectivement par les angles $\theta$ et $\varphi$ (voir figure 1). Cela permet de définir les constantes géométriques suivantes :

$$
A=\frac{\tan \theta \tan \varphi}{\tan \theta+\tan \varphi}, B=\frac{\tan \varphi}{\tan \theta+\tan \varphi} \text { et } C=\frac{\tan \theta}{\tan \theta+\tan \varphi}
$$

Dans la suite $A, B$ et $C$ sont fixées respectivement à $1 / 10,4 / 5$ et $1 / 5$, ce qui correspond à des angles $\theta$ et $\varphi$ respectivement de $7^{\circ}$ et $26^{\circ}$.

On appellera $x$ la coordonnée selon la direction du vent et $y$ celle selon la direction perpendiculaire. Une coupe transversale de dune est alors complètement décrite par les coordonnées de son sommet $(x, h)$ (voir la figure 1$)$. 


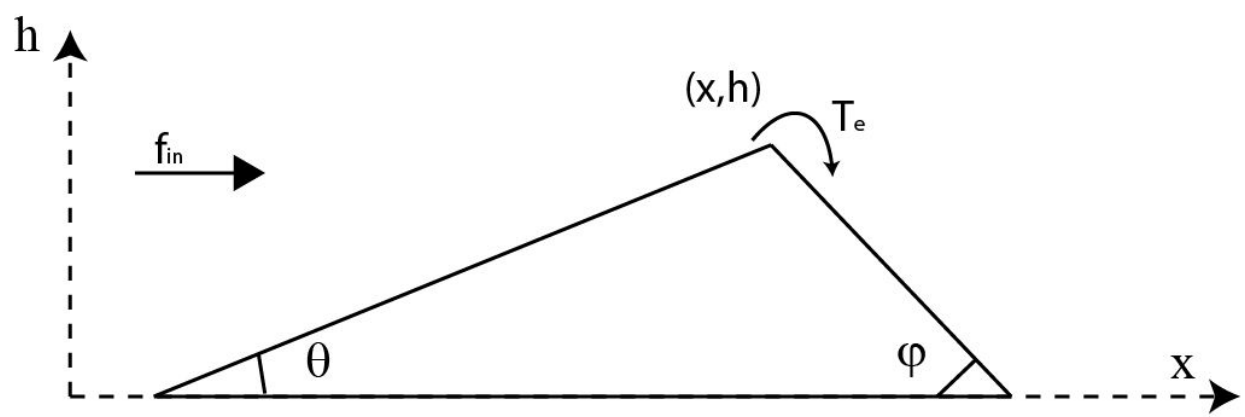

Figure 1. Schéma d'une coupe transverse de dune. Les angles des faces amont et aval avec l'horizontale sont respectivement $\theta$ et $\varphi$. Le sommet de la dune est repéré par ses coordonnées $x$ et h. Un flux de sédiment $f_{\text {in }}$ est imposé et le taux de capture du sédiment par la face aval est $T_{e}$.

La force de l'écoulement détermine le flux de sédiment $q$ au sommet de la dune. Le sédiment entraîné par l'écoulement au sommet de la dune peut être capté par la face aval de la dune. La probabilité de capture est définie par le taux de capture $T_{e}$. Pour une force d'écoulement donnée, le taux de capture est supposé être fonction de la hauteur de la dune uniquement. Un flux de sédiment $f_{\text {in }}$ constant est imposé en amont de la dune.

\subsection{Mise en équation}

En écrivant la conservation de la matière sur une dune pour la face aval et la section complète, on obtient le système d'équations suivant :

$h \frac{\partial x}{\partial t}=q\left(B T_{e}+C\right)-C f_{i n}-B \frac{\partial J_{d}}{\partial y}+C \frac{\partial J_{u}}{\partial y}$,

$h \frac{\partial h}{\partial t}=A\left[q\left(T_{e}-1\right)+f_{i n}-\frac{\partial J_{d}}{\partial y}-\frac{\partial J_{u}}{\partial y}\right]$

où $J_{u}$ et $J_{d}$ sont les flux latéraux respectivement sur les faces amont et aval de la dune. En considérant que les flux latéraux sur les faces aval et amont sont proportionnels aux aires sur lesquelles les faces respectives de deux sections successives se chevauchent (NIIYA et al., 2010), on peut écrire que :

$$
\begin{aligned}
& J_{u}=D_{u}\left(-\frac{B}{A} h \frac{\partial h}{\partial y}+h \frac{\partial x}{\partial y}\right), \\
& J_{d}=D_{d}\left(-\frac{C}{A} h \frac{\partial h}{\partial y}-h \frac{\partial x}{\partial y}\right) .
\end{aligned}
$$

où $D_{u}$ et $D_{d}$ sont des constantes homogènes à des coefficients de diffusion et caractérisent l'intensité de la diffusion latérale respectivement sur la face amont et aval. Le système d'équations (2) et (3) complété par les relations (4) et (5) caractérise complètement la dynamique de notre dune transverse. 


\section{Analyse de stabilité linéaire d'une dune transverse}

Le système ci-dessus possède une solution stationnaire uniforme qui correspond à une dune transverse rectiligne se mouvant à vitesse constante. On cherche à savoir si cette solution est stable vis-à-vis de perturbations quelconques. C'est le principe de l'analyse de stabilité linéaire. Celle-ci consiste plus précisément à perturber le système par un signal sinusoïdal de nombre d'onde $k$ et de calculer son taux de croissance $\omega$. Si le taux de croissance est négatif pour tous les nombres d'onde, toutes les perturbations vont s'atténuer dans le temps. La solution sera stable. Au contraire, si on trouve au moins un nombre d'onde dont le taux de croissance est positif, celui va croître exponentiellement. La solution est alors instable. C'est en général le mode instable qui possède le plus grand taux de croissance qui va se développer et dominer sur tous les autres.

\subsection{Solution homogène}

On cherche une solution homogène de la forme $x=V_{0} t$ et $h=h_{0}$, où $V_{0}$ la vitesse d'avancée et $h_{0}$ la hauteur de la dune sont constantes. En injectant ces relations dans les équations (2) et (3), on trouve :

$h_{0} V_{0}=q T_{e}\left(h_{0}\right)$,

$f_{\text {in }}=q\left(1-T_{e}\left(h_{0}\right)\right)$.

Cette solution représente une dune transverse de hauteur uniforme qui avance à vitesse constante. Plus la dune est haute, plus elle avance lentement.

\subsection{Etude de stabilité linéaire}

La solution homogène est perturbée par une composante sinusoïdale de la forme suivante :

$$
\begin{aligned}
& x(y, t)=x_{0}(t)+x_{1}(y, t) \\
& h(y, t)=h_{0}+h_{1}(y, t)
\end{aligned}
$$

où :

$$
\begin{aligned}
& x_{1}(y, t)=x_{10} e^{\omega t+i k y} \\
& h_{1}(y, t)=h_{10} e^{\omega t+i k y}
\end{aligned}
$$

où $k$ est le nombre d'onde de la perturbation et $\omega$ son taux de croissance.

En injectant (8) et (9) dans le système (2) et (3) et en ne retenant que les termes linéaires en $h_{1}$ et $x_{1}$, on obtient une expression pour le taux de croissance $\omega$ en fonction de $k$ :

$$
\begin{aligned}
& 2 h_{0}^{2} \omega_{ \pm}=A q \delta T_{e}\left(h_{0}\right)-(1+\rho) D_{u} h_{0}^{2} k^{2} \\
& \pm \sqrt{A^{2} q^{2} \delta^{2} T_{e}\left(h_{0}\right)^{2}+2 A q(1-\rho)(\delta-2) T_{e}\left(h_{0}\right) D_{u} h_{0}^{2} k^{2}+(1-\rho)^{2} D_{u}^{2} h_{0}^{4} k^{4}}
\end{aligned}
$$

où :

$$
\delta=\left.\frac{h_{0}}{T_{e}\left(h_{0}\right)} \frac{d T_{e}}{d h}\right|_{h_{0}} \text { et } \rho=\frac{D_{d}}{D_{u}}
$$




\section{XII ${ }^{\text {èmes }}$ Journées Nationales Génie Côtier - Génie Civil \\ Cherbourg, 12-14 juin 2012}

Le paramètre $\delta$ est la variation relative du taux de capture de sédiment sur la face aval. Si la diffusion est négligée, pour un $\delta$ positif, on s'attend à ce que la dune soit déstabilisée. En effet, si la hauteur de la dune augmente, elle captera plus de sédiment donc aura tendance à poursuivre sa croissance, l'effet sera donc déstabilisant. Au contraire un $\delta$ négatif aura l'effet inverse, la dune sera donc stable.

Le paramètre $\rho$ est le rapport des coefficients de diffusion sur les faces aval et amont de la dune. Le coefficient de diffusion de la face amont aura un effet stabilisant alors que celui de la face aval aura un effet déstabilisant. Pour une dune de hauteur uniforme, considérons deux tranches légèrement décalées selon $x$. Sous l'effet de la diffusion sur la face amont, la tranche en amont va céder du sédiment à celle en aval. La tranche en aval va donc croître. Une tranche qui croît voit sa vitesse diminuer, la dune amont va donc rattraper celle qui était en aval. Les tranches vont se trouver alignées, l'effet de la diffusion amont est bien stabilisant pour la dune transverse. Au contraire, la diffusion sur la face aval aura l'effet inverse et déstabilisera la dune transverse rectiligne. Donc plus $\rho$ sera élevé, plus l'effet de la diffusion sera déstabilisant.

Par la suite, on s'intéressera uniquement au mode le plus instable $\omega_{+}$, et plus précisément à sa partie réelle. Analysons dans un premier temps le comportement de $\omega_{+}$ à grand $k$ et à petit $k$.

A grand $k$, la diffusion domine et a un effet stabilisant. Les perturbations de petite longueur d'onde vont donc s'atténuer dans le temps $\left[\omega_{+} \approx-D k^{2}\right.$ où $\left.D=\max \left(D_{u}, D_{d}\right)\right]$.

On cherche à savoir maintenant s'il existe des modes instables pour des valeurs finies du nombre d'onde. Pour cela, on effectue le développement du taux de croissance à petit nombre d'onde. Il apparaît 2 situations différentes selon le signe de $\delta$ :

(i) $\delta>0$

$\omega_{+} \approx \frac{A q \delta T_{e}\left(h_{0}\right)}{h_{0}^{2}}-D_{u}\left[\rho+\frac{1-\rho}{\delta}\right] k^{2}$

(ii) $\delta<0$

$\omega_{+} \approx-D_{u}\left[1+\frac{\rho-1}{\delta}\right] k^{2}$

Il apparaît que la présence de modes instables dépend uniquement des deux paramètres $\rho$ et $\delta$.

On trouve que dans le cas où $\delta>0$, il apparaît une bande de vecteur d'onde instable quelque soit la valeur du paramètre $\rho$. La dune transverse sera donc toujours instable. Dans le cas opposé (i.e., $\delta<0$ ), il n'existe de modes instables que si $\rho$ est une supérieur à valeur critique $\rho_{c}=1-\delta$. La dune transverse sera donc stable pour $\rho<\rho_{c}$. 


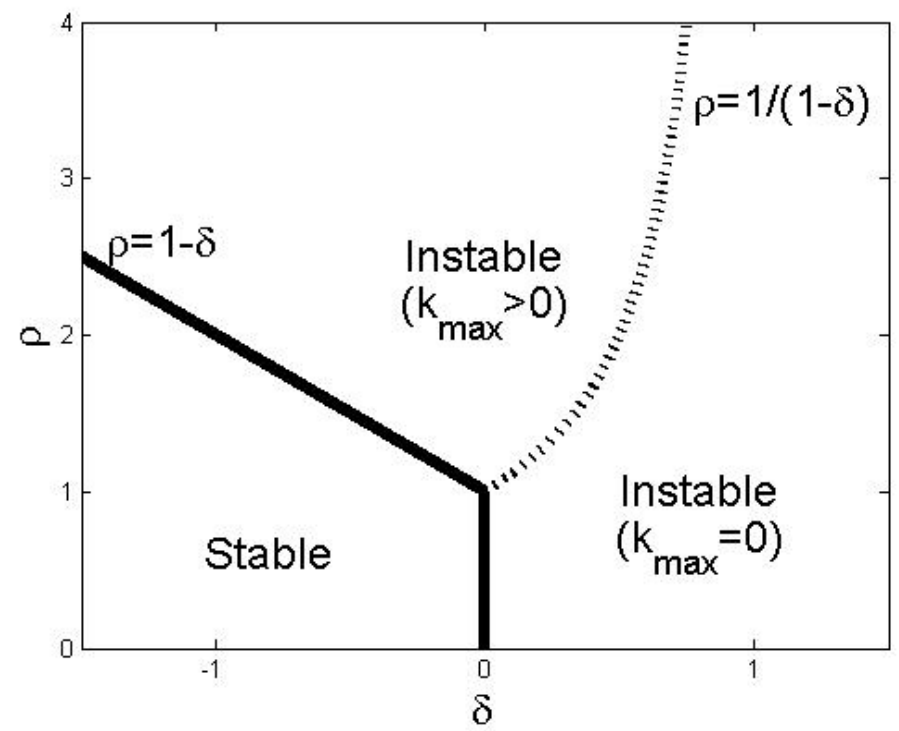

Figure 2. Domaine de stabilité d'une dune transverse rectiligne dans l'espace des paramètres $\delta$ et $\rho$. Pour $\delta>0$, une dune transverse rectiligne est toujours instable, alors que pour $\delta<0$, elle est stable si $\rho<1-\delta$.

La figure 2 résume nos résultats en montrant le domaine de stabilité des dunes transverses dans l'espace des paramètres $\delta$ et $\rho$. Dans le domaine d'instabilité de la dune, deux scénarii différents sont attendus selon que le mode le plus instable $k_{\max }$ correspond à un nombre d'onde fini ou non (voir figure 3). Dans le cas où $k_{\max }=0$, on s'attend à ce que la dune subisse une augmentation ou une diminution uniforme de sa hauteur, tandis que dans le cas où $k_{\max }>0$, la hauteur et la ligne de crête développeront une ondulation de longueur d'onde $2 \pi / k_{\max }$. Dans ce dernier cas, l'amplitude de l'ondulation croîtra dans un premier temps puis pourra éventuellement saturer à une valeur finie sous l'effet des non-linéarités (non prises en compte dans cette analyse linéaire) ou augmenter exagérément pour conduire à une fragmentation de la dune en une multitude de dunes plus petites.

\section{Discussion}

\subsection{Taux de capture}

La variation du taux de capture avec de la hauteur de la dune joue un rôle prépondérant sur la stabilité de la dune transverse. Il est possible d’avoir une idée de sa variation à partir de considérations physiques élémentaires. Le sédiment au sommet de la dune est soumis à une force de cisaillement produite par l'écoulement, ce qui engendre un certain flux $q$ au sommet de la dune. Le sédiment arrivant au sommet de la dune sera projeté à une certaine distance en aval. Cette distance dépend essentiellement de la force de l'écoulement. Par ailleurs, plus la dune est haute, plus sa face d'avalanche est étendue, 


\section{XII ${ }^{\text {èmes }}$ Journées Nationales Génie Côtier - Génie Civil \\ Cherbourg, 12-14 juin 2012}

et plus la probabilité pour que le sédiment soit capté par celle-ci est importante. Pour un écoulement d'intensité donné, on s'attend donc à ce que le taux de capture augmente de façon monotone (i.e., $\delta>0$ ) puis sature $(\delta=0)$ à partir d'une valeur critique de $h$. Il apparaît donc que seules les situations où $\delta$ est positif ou nul semblent réalistes.

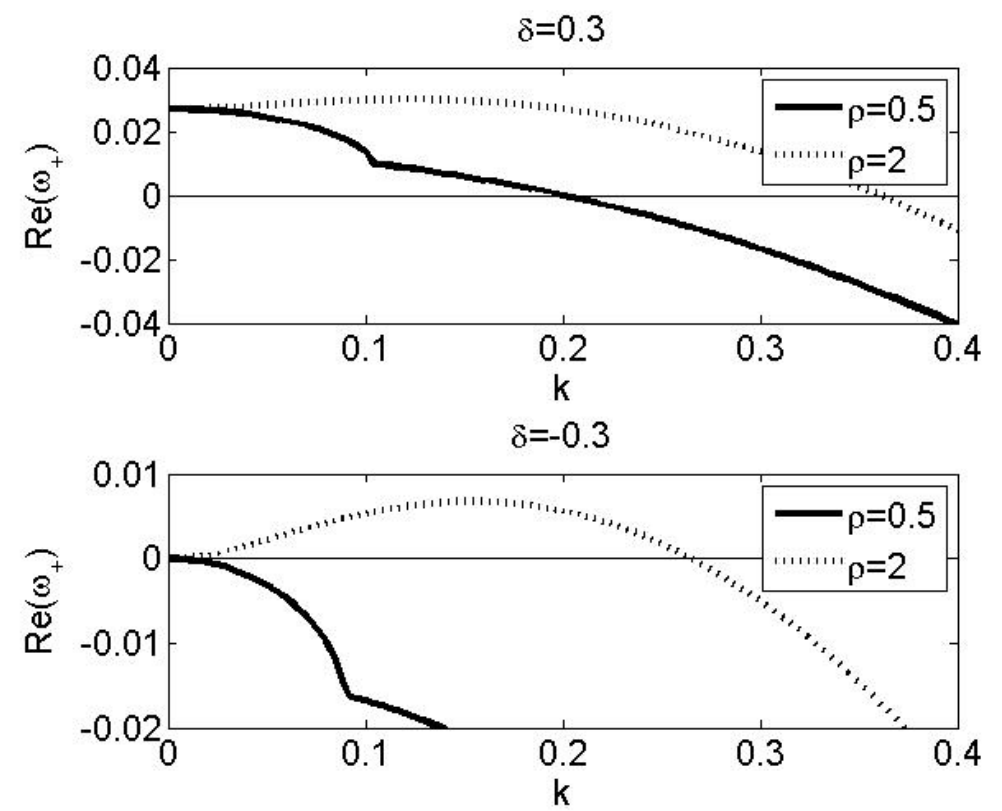

Figure 3. Diagrammes de dispersion pour deux valeurs de $\rho$ et de $\delta\left(q / D_{u}=0.5\right.$;

$T e=0.9)$. Pour $\delta=0.3$, $\omega$ est positif sur une bande de $k$, la dune transverse est donc instable pour les deux valeurs de $\rho$. Pour $\delta=-0.3$, la dune transverse est instable dans le cas $\rho=0.5$, et stable pour $\rho=2$.

\subsection{Coefficients de diffusion}

Le rapport $\rho$ entre les coefficients de diffusion latéraux $D_{u}$ et $D_{d}$ joue aussi un grand rôle dans la détermination de la stabilité. Plusieurs causes peuvent produire des flux latéraux (HERSEN, 2004). Tout d'abord la composante latérale de l'écoulement, lorsque la forme de la dune n'est plus invariante selon la direction transverse, contribue à distribuer les grains latéralement. Ensuite les avalanches qui se produisent sur la face aval ont une certaine étendue latérale, ce qui contribue au flux de diffusion $J_{d}$. Certains grains se déplacent en saltation et créent un impact qui met d'autres grains en mouvement. Le mouvement de ces grains est alors sensible à la pente locale autour d'un grain, qui n'est pas nécessairement la pente calculée dans la direction principale de l'écoulement. Cela cause des flux latéraux sur les faces amont. Peu de mesures existent sur la quantification de ces flux et il est donc difficile d'en donner des estimations. Il serait donc intéressant de les quantifier précisément par des mesures de terrain ou des expériences modèles en laboratoire. 


\subsection{Validation du modèle}

Parteli et al. ont étudié la stabilité des dunes transverses à partir d'une simulation numérique basée sur la résolution des équations hydrodynamiques couplées à un modèle de transport de sédiment (PARTELI et al., 2011). Cette étude a montré que les dunes transverses rectilignes sont intrinsèquement instables. C'est ce que prédit notre modèle dans le cas où $T^{\prime}{ }^{\prime}>0$.

\section{Conclusion et perspectives}

Une analyse de stabilité linéaire de dunes transverses a été menée grâce à un modèle simplifié qui prend en compte les principaux ingrédients du transport sédimentaire. Nous prédisons le domaine de stabilité des dunes transverses et montrons que seuls deux paramètres jouent un rôle pertinent: le taux de capture et le rapport des coefficients de diffusion sur les faces amont et aval.

La prise en compte des effets non-linéaires reste à faire pour déterminer l'évolution des dunes transverses quand elles sont instables. Ceci peut être réalisé simplement en intégrant numériquement notre système d'équations, ce que nous envisageons de faire à court terme. On peut néanmoins déjà prévoir les différents scenarii attendus. Dans un premier temps, la hauteur et la ligne de crête vont subir une ondulation à une longueur d'onde bien définie, dont l'amplitude croît dans le temps. Puis on verra soit une stabilisation de l'amplitude, soit une croissance indéfinie qui produira une fragmentation de la dune en une multitude de petites dunes.

Cette étude a été partiellement financée par le Ministère de la Défense (Direction Générale de l’Armement) et la Région Bretagne.

\section{Références bibliographiques}

HERSEN P. (2004). On the crescentic shape of barchan dunes. The European Physical Journal B-Condensed matter and Complex Systems, Vol. 37, Number 4, pp 507-514. KROY K., SAUERMANN G., HERRMANN H.J. (2002). Minimal Model for sand dune. Physical Review Letters, Vol. 88, 054301. doi:10.1103/PhysRevLett.88.054301

NIIYA H., AWAZU A., NISHIMORI H. (2010). Three-Dimensional Dune Skeleton Model as a Coupled Dynamical System of Two-Dimensional Cross Sections. Journal of the Physical Society of Japan, Vol. 79, No. 6, June, 2010, 063002. doi:10.1143/JPSJ.79.063002 PARTELI E., ANDRADE J., HERRMANN H., (2011). Transverse Instability of Dunes. Physical Review Letters, Vol. 107, 188001. doi:10.1103/PhysRevLett.107.188001

RICHARDS K.J. (1980). The formation of ripples and dunes on an erodible bed. J. Fluid Mech., Vol. 99, 597. doi:10.1017/S002211208000078X

VALANCE A. (2005). Formation of ripples over sand bed submitted to a turbulent shear flow. Eur. Phys. J. B., Vol. 45, pp 433-442. doi:10.1140/epjb/e2005-00201-9 